\title{
Infections of implantable cardiac devices by biofilm forming bacteria in western Algeria hospitals
}

\author{
${ }^{1}$ Meziani, Z., ${ }^{1}$ Hassaine, H., and ${ }^{2}$ Belhachemi, F. \\ ${ }^{1}$ Laboratory of Applied Microbiology in Food, Biomedical and Environment (LAMAABE), \\ University of Tlemcen, Algeria \\ ${ }^{2}$ Department of Cardiology, Hospital of Tlemcen, Algeria \\ *Correspondence to: zahera_fd@yahoo.fr; 00213553406801
}

\begin{abstract}
:
Background: The significant increase in the use of implantable cardiac devices (ICDs) has been accompanied by biofilm formation and increase rate of infection on the devices. The purpose of our study is to describe the clinical and microbiological findings of infection of ICDs in the cardiology units of western Algeria hospitals. Methodology: All patients with clinical diagnosis of ICD infections or infective endocarditis upon removal of their ICDs from December 2012 to August 2014 in cardiology units of 4 Algerian hospitals were included in the study. Each element of the ICD pocket and lead was separately sonicated in sterile saline, inoculated onto Chapman and MacConkey agar plates and incubated aerobically at $37^{\circ} \mathrm{C}$ for colony count after 24 hours. Biochemical identification of the bacteria isolates was made by API 20E, API 20 NE and API Staph, and confirmed by Siemens Healthcare Diagnostics WalkAway ${ }^{\circledR} 96$ Plus System. Antibiotic susceptibility testing on each isolate was performed by the disk diffusion method on Mueller Hinton agar. Biofilm formation was detected by Congo Red Agar (CRA) and Tissue Culture Plate (TCP) methods, and hydrophobicity of the bacterial cell was determined by the MATH protocol.

Results: Over a period of twenty-one months, 17 ICDs were removed from patients with post-operative infections; $6(35.3 \%)$ had early infection of ICD and $11(64.7 \%)$ had late ICD infection. Fifty-four bacterial strains were isolated and identified, with coagulase-negative staphylococci being the predominant bacteria with $46.3 \%(25 / 54)$. There was no significant association between hydrophobicity and antimicrobial resistance in the 54 isolates but there is positive correlation between biofilm production and antimicrobial resistance, with the strongest biofilm producers resistant to more than one antibiotic. Four independent predictors of infection of resynchronization devices were reported; reoperation, multi-morbidity, long procedure, and ICD implantation. Conclusion: Our study is the first in Algeria to describe microbiological characteristics of ICD infection. The bacteria in the biofilm were protected, more resistant and tolerated high concentrations of antibiotics and thus played a major role in the development of ICD infections. Despite the improvements in ICD design and implantation techniques, ICD infection remains a serious challenge.
\end{abstract}

Keywords: implantable cardiac devices, staphylococci, resistance, biofilm, hydrophobicity

Received January 28, 2020; Revised May 4, 2020; Accepted May 6, 2020

Copyright 2020 AJCEM Open Access. This article is licensed and distributed under the terms of the Creative Commons Attrition 4.0 International License <a rel="license" href="http://creativecommons.org/licenses/by/4.0/", which permits unrestricted use, distribution and reproduction in any medium, provided credit is given to the original author(s) and the source.

\section{Infections des dispositifs cardiaques implantables par des bactéries formant un biofilm dans les hôpitaux de l'ouest Algérien}

\author{
${ }^{1}$ Meziani, Z., ${ }^{1}$ Hassaine, $H_{\text {., }}$ et ${ }^{2}$ Belhachemi, F. \\ ${ }^{1}$ Laboratoire de microbiologie appliquée à l'alimentation, la biomédecine et l'environnement (LAMAABE), \\ Université de Tlemcen, Algérie
}




\author{
2Département de cardiologie, Hôpital de Tlemcen, Algérie \\ *Correspondance avec: zahera_fd@yahoo.fr; 00213553406801
}

\title{
Résumé:
}

Contexte: L'augmentation significative de l'utilisation des dispositifs cardiaques implantables est un risque majeur d'augmentation du taux d'infection et donc du risque de formation d'un biofilm sur ce genre de dispositifs. L'objectif de notre étude est de décrire les résultats cliniques et microbiologiques de l'infection sur les dispositifs cardiaques implantables (DCI) dans les unités de cardiologie des hôpitaux de l'ouest Algérien. Méthodologie: Tous les patients cliniquement diagnostiqués avec une infection sur DCI, ou une endocardite infectieuse et ayant subit un retrait de leur dispositif cardiaque sont inclus dans cette étude et cela sur une période entre décembre 2012 et aout 2014 dans 4 unités de cardiologie. Chaque élément du DCI (boitier et sonde) est trempé séparément dans une solution saline stérile, ensemencé sur deux milieux de culture, un milieu de Chapman et un milieu MacConkey et incubé en aérobiose à $37^{\circ} \mathrm{C}$ pour la numération des colonies après 24 heures. L'identification biochimique des isolats de bactéries est effectuée par le API 20E, API 20 NE et API Staph, et confirmée par le système WalkAway ${ }^{\circledR} 96$ Plus de Siemens Healthcare Diagnostics. Les tests de sensibilité aux antibiotiques de chaque isolat sont effectués par la méthode de diffusion des disques sur gélose de Mueller Hinton. La formation d'un biofilm est détectée par les méthodes de la gélose rouge du Congo (CRA) et de la plaque de culture tissulaire (TCP), et l'hydrophobicité de la cellule bactérienne est déterminée par le protocole MATH.

Résultats: Sur une période de 21 mois, 17 DCI sont retirés de patients atteints d'infections postopératoires; 6 patients $(35,3 \%)$ sont identifiés comme ayant une infection précoce sur leurs DCI et 11 patients $(64,7 \%)$ ayant une infection tardive. Cinquante-quatre souches bactériennes sont isolées et identifiées, les staphylocoques à coagulase négative étant les bactéries prédominantes avec $46,3 \%(25 / 54)$. Il n'y a pas d'association significative entre l'hydrophobicité et la résistance aux antimicrobiens dans les 54 isolats, mais il existe une corrélation positive entre la production de biofilm et la résistance aux antimicrobiens, les plus puissants en biofilm sont résistant à plus d'un antibiotique. Quatre facteurs prédictifs indépendants d'infection des dispositifs cardiaques implantable sont retrouvés dans ce travail: ré-intervention, longue procédure, sujets multi-tarés, et implantation d'un DCI

Conclusion: Notre étude est la première en Algérie à décrire les caractéristiques microbiologiques de l'infection des DCI. Les bactéries présentes dans le biofilm sont protégées, plus résistantes et tolèrent de fortes concentrations d'antibiotiques et jouent ainsi un rôle majeur dans le développement des infections par DCI. Malgré des améliorations dans les techniques de conception et d'implantation de DCI, l'infection des dispositifs cardiaques implantables reste un problème grave et très couteux.

Mots-clés: dispositifs cardiaques implantables; staphylocoque; résistance; biofilm; hydrophobicité

\section{Introduction:}

The permanent implantable cardiac device (ICD) is one of the most widely used cardiac rhythm control devices. Since their introduction in the early 1980s, ICD have become a life-saving therapeutic tool for patients with ventricular arrhythmia. Virtually all the countries surveyed showed significant rise in the use of ICD, the largest implanter being the USA with 434 new implants per million populations (1). Despite improvements in cardiac device design, application of timely infection control practices, and administration of antibiotic prophylaxis at the time of device placement, infections continue to be observed. The infection rate is highly variable, ranging from 0.5 to $12 \%(2,3)$. Infection of implantable cardiac device (ICD) may be local, limited to the pulse generator pocket and/or the subcutaneous portion of the leads, or systemic, involving the transvenous intravascular electrode (4). Several factors have been reported to be associated with a greater risk of ICD infections and these include; immunosuppression (e.g. renal dysfunction and corti- costeroid use); oral anticoagulation use; coexisting illnesses; periprocedural factors including failure to administer perioperative antimicrobial prophylaxis; device revision/replacement; and operators' experience (5). Because of the invasiveness, these devices have multiple comorbidities in ICD recipients, the benefits of which can be overshadowed by infectious complications. Infection of ICD is usually a serious and fearsome complication requiring both complete removal of the infected device and systemic antimicrobial treatment $(5,6)$. Although the implantation of pacemakers is a procedure characterized by low rate of complication, infections, mainly of the pacemaker generator pocket, is one of the most common complications with incidence ranging from 1 to $5 \%$. Despite the low incidence, such infections have a worrying development, mostly because it is associated with high morbidity and potential fatality (7).

Microbiological diagnosis of ICD infection is of great importance for appropriate treatment. Staphylococcal species including Staphylococcus aureus and coagulase negative staphylococci (CoNS) represent the 
majority of bacterial agents of ICD infection $(8,9)$. However, some unusual microorganisms such as Propionibacterium spp., Corynebacterium spp., Acinetobacter baumannii, and Haemophilus influenzae may also be involved and antibiotic resistance is often detected in them $(10,11)$. Furthermore, one major concern among professionals and bacteriologists is the risk of biofilm formation on the ICDs which consequently favors infection. In recent decades, biofilm formations have been widely reported to be responsible for nosocomial infections especially of prosthetic implants, tubes, leads and catheters $(12,13)$.

It is speculated that the presence of an ICD is conducive for microbial colonization contributing greatly to the development of biofilms, which in turn, explains the occurrence of endocarditis $(14,15)$. It is interesting to note that biofilm consists of a structured medium of microbial cells that adhere to a solid surface and is surrounded by a matrix of extracellular polymeric substances. This microbial combination is a form of protection encouraging symbiotic relationships, tolerance, as well as antimicrobial resistance (16).

The goal of this study is to first describe the bacteriological characteristics of ICD infections over a period of 2 years in four cardiology units of one private and three university hospital centers in western Algeria, and secondly to determine whether the isolated bacteria have the ability to form biofilms that may be responsible for triggering infection, especially in late stage endocarditis.

\section{Materials and method:}

\section{Study setting:}

This study is carried out at the Laboratory of Microbiology Applied to Food, Biomedical and Environment (LAMAABE) of the University Abou-Bekr Bekaid-Tlemcen, Algeria.

\section{Subjects}

All the patients with clinical diagnosis of ICD infection or infective endocarditis upon removal of their cardiac devices from December 2012 to August 2014 in four cardiology units of three hospitals in Algeria were included in this study. The clinical diagnosis of ICD infection was made by the clinician based on local signs of inflammation such as erythema, heat, redness or purulent drainage, outward displacement of the pocket, septicemia or infectious endocarditis of the heart. The time of infection was recorded and ICD infection was categorized as; 'very early' if infection occurs 1 month after the latest ICD procedure; 'early' if infection occur between 2 and 12 months of the latest procedure, and 'late' if infection occurs 12 months after the latest procedure.

\section{Ethical approval}

Ethical clearance was obtained from the Institutional Review Board at the Faculty of Health Science of the University of Algeria. The administrative authorizations were obtained from the Delegation of Public Health of the Regional Hospitals of Western Algeria. Moreover, written informed consent was obtained from all study patients prior to interview and DCI collection

Collection of demographic and clinical data Demographic and clinical data collected from each patient by direct interview included age, gender, existence of an underlying anterior cardiopathy or a history of infective endocarditis, existence of diabetes, immunosuppression, primary implantation or change of pocket, antibiotic therapy and possible infection or surgery programmed during the last five years.

\section{Microbiological culture of the ICDs}

After removal by the surgeons, the implantable cardiac devices (ICDs) were placed in sterile boxes and transported to the microbiology laboratory within 30 minutes. Each element of the ICD pocket and lead was soaked separately in sterile saline solution and sonicated for 5 minutes at a frequency of $20 \mathrm{Khz}$, and vortexed for $30 \mathrm{sec}$ to detach and detect sessile bacteria hanging on to these devices $(17,18)$. After sonication, $100 \mu \mathrm{L}$ of the soaking liquid was inoculated onto Chapman agar and MacConkey agar plates. The number of CFU/mL was counted after 24 hours aerobic incubation. The minimum detection threshold was $2 \mathrm{CFU} / \mathrm{mL}$ (19).

\section{Biochemical identification and susceptibility testing of bacteria isolates}

The biochemical identification of the bacteria isolates was done using the API $20 \mathrm{E}$ and API 20NE Staph API (BioMérieux, Marcy l'Etoile, France), and confirmed by the Siemens Healthcare Diagnostics WalkAway ${ }^{\circledR}$ 96 Plus System. The antibiotic susceptibility and resistance phenotypes were determined by the disk diffusion method on MuellerHinton agar (Biorad, Marnes-la-Coquette, France) according to the recommendations of the antibiogram committee of the French Society of Microbiology (20). The antibiotic disks used were penicillin (10iu), oxacillin $(5 \mu \mathrm{g})$, cefoxitin $(30 \mu \mathrm{g})$, gentamicin $(10 \mu \mathrm{g})$, 
tobramycin $(10 \mu \mathrm{g})$, amikacin $(30 \mu \mathrm{g})$, vancomycin $(30 \mu \mathrm{g})$, rifampin $(30 \mu \mathrm{g})$, fosfomycin $(50 \mu \mathrm{g})$, fusidic acid $(10 \mu \mathrm{g})$, clindamycin (2 $\mu \mathrm{g})$, pristinamycin $(15 \mu \mathrm{g})$, erythromycin (15 $\mu \mathrm{g})$, ofloxacin $(5 \mu \mathrm{g})$, tetracycline $(30 \mu \mathrm{g})$, chloramphenicol $(30 \mu \mathrm{g})$, imipenem $(10 \mu \mathrm{g})$, and trimethoprim/sulfamethoxazole $(25 \mu \mathrm{g})$, cefotaxime $(5 \mu \mathrm{g})$, ceftriaxone $(30 \mu \mathrm{g})$, nalidixic acid $(30 \mu \mathrm{g})$, ciprofloxacin $(5 \mu \mathrm{g})$, amoxicillin $(20 \mu \mathrm{g})$, and clavulanic acid $(10 \mu \mathrm{g})$.

\section{Detection of biofilm by Tissue Culture Plate (TCP) method}

Quantitative measurement of biofilm production in the isolates was done using a microtiter assay. In brief, cells were grown overnight in Brain Heart Infusion Broth (BHIB). The broth was then diluted 1:100 and inoculated into microtiter plates. After 24 hours incubation at $37^{\circ} \mathrm{C}$, the plates were washed, stained with crystal violet, and the optical density measured at $570 \mathrm{~nm}$ (20). Each test was performed in triplicate. As a negative control, the uninoculated medium was used to determine the initial OD. Mean OD values were calculated for all tested strains and the negative control.

The threshold value (ODC) was established as three standard deviations (SD) above the mean OD of the negative control $i$. e. $O D C=$ mean $O D$ of the negative control + ( $3 \times$ SD of negative control). The ODc value was calculated separately for each microtiter plate. When a negative value was obtained, it was presented as zero, while any positive value indicated the production of biofilms. For the interpretation of the results, the classification of the obtained results was based on the control OD. The strains are classified as follows: $O D \leq D O t$ (control) is non-biofilm forming; DOt $\times 2 \leq \mathrm{OD} \leq \mathrm{DOt} \times 4$ is moderate biofilm-forming, and DOt $\times 4 \leq O D$ is highly (strong) biofilm-forming (21).

\section{Detection of biofilm by Congo Red Agar (CRA) method}

The Congo Red Agar method was used for detecting the ability of isolate to produce slime. The CRA medium was prepared with $37 \mathrm{~g} / \mathrm{L}$ BHI broth, $50 \mathrm{~g} / \mathrm{L}$ sucrose, $10 \mathrm{~g} / \mathrm{L}$ agar, and $0.8 \mathrm{~g} / \mathrm{L}$ Congo Red. The Congo Red stain was prepared as a concentrated aqueous solution and autoclaved at $121^{\circ} \mathrm{C}$ for 15 min separately from other medium constituents, and was then added when the agar had cooled to $55^{\circ} \mathrm{C}$. The plates were inoculated with bacterial isolate and incubated at $37^{\circ} \mathrm{C}$ for 24 hours. A positive result was indicated by black colonies while non-producing strains developed red colonies (22). For the evaluation of colonies colors, a four-color reference scale was used according to Satorres and Alcaráz (23). Black and almost black burgundy were classified as biofilm producers, while burgundy and red as non-biofilm producing strains. This test was performed in triplicate

\section{Hydrophobicity assays}

The hydrophobicity of the bacterial wall was evaluated with the MATH protocol (24) using hexadecane as a solvent. The bacteria strains were first grown in $50 \mathrm{~mL}$ of Luria Bertani (LB) and incubated for 18 hours at $37^{\circ} \mathrm{C}$. The cells were recuperated by centrifugation at $5000 \mathrm{rpm}$ for $15 \mathrm{~min}$. The pellet obtained was then washed after two successive centrifugations with PBS (Phosphate Buffered Saline $\mathrm{pH}$ 7.1) and suspended in the same buffer at an initial optical density (ODi) between 0.8 and 1 at $600 \mathrm{~nm}$. A volume of $0.3 \mathrm{ml}$ of each solvent was added to $1.8 \mathrm{ml}$ of bacterial suspension and the mixture was vortexed for $2 \mathrm{~min}$. After a $20 \mathrm{~min}$ settling, the optical density (ODf) of the aqueous phase was measured at $600 \mathrm{~nm}$ and the percentage of adhesion to solvent was then calculated using the following equation; $\mathrm{CSH}$ $\%=[(\mathrm{ODi}-\mathrm{ODf}) / \mathrm{ODi}] \times 100$ (25). A CSH of $0-$ $20 \%$ is defined as weak, $21-50 \%$ as moderate and $>40 \%$ as strong hydrophobicity.

\section{Results:}

From December 2012 to August 2014, a total of 315 implantable cardiac devices (ICDs) were implanted by the cardiology units of the four hospitals, out of which ICD was removed in 17 patients, all of whom developed infection on the ICDs, giving an infection rate of $5.4 \%$. All 17 patients underwent only one surgical procedure except for one patient who had two surgical procedures within three months due to repeated infections of his ICD. The age range of the patients was 60 to 75 years; 13 $(76.4 \%)$ were male and $5(29.4 \%)$ were diabetic.

All the patients were on antibiotic therapy on the day of cardiac device insertion. Four independent predictive factors of ICD infection identified were; reintervention, long procedure, multi-morbidity, and ICD implantation procedure. Six patients $(35.3 \%)$ had early infection of ICD while 11 patients $(64.7 \%)$ had late infection. There were 9 patients with associated primary ICD implantation infections while there were 8 with change of pocket ICD infection (Table $1)$. 
Table 1: Distribution of bacteria strains according to the implantable cardiac device (ICD)

\begin{tabular}{|c|c|c|c|c|c|c|c|}
\hline \multirow[t]{2}{*}{ Pacemaker } & \multicolumn{2}{|c|}{ Isolated strains } & \multirow[t]{2}{*}{ Hospital } & \multirow{2}{*}{$\begin{array}{l}\text { Gender } \\
(M / F)\end{array}$} & \multirow{2}{*}{$\begin{array}{l}\text { Duration of the } \\
\text { intervention }\end{array}$} & \multirow{2}{*}{$\begin{array}{c}\text { Primo or } \\
\text { change of } \\
\text { pocket }(\mathrm{P} / \mathrm{C})\end{array}$} & \multirow[t]{2}{*}{ Endocarditis } \\
\hline & Pocket & Lead & & & & & \\
\hline \multirow[t]{2}{*}{ P1 } & Negative & S1: Moraxella & $\mathrm{CHU}$ Tlemcen & $\mathrm{F}$ & $50 \mathrm{~min}$ & $\mathrm{P}$ & \\
\hline & & S'1: Staphylococcus xylosus & & & & & \\
\hline P2 & P'2: Staphylococcus xylosus & Negative & CHU Tlemcen & M & $1 \mathrm{~h} 20 \mathrm{~min}$ & c & \\
\hline \multirow[t]{2}{*}{ P3 (DAI) } & P3: Pseudomonas aeruginosa & S3: Enterobacter cloacae & $\begin{array}{l}\text { EH Ain } \\
\text { Temouchent }\end{array}$ & M & $7 \mathrm{~h}$ & $\mathrm{P}$ & \\
\hline & & S3p: Pseudomonas aeruginosa & & & & & \\
\hline P4 & P'4: Staphylococcus épidermidis & $\begin{array}{l}\text { S'4: Staphylococcus } \\
\text { épidermidis }\end{array}$ & $\begin{array}{l}\text { EH Ain } \\
\text { Temouchent }\end{array}$ & M & 4hs & $P$ & \\
\hline P5 & P'5: Staphylococcus sciuri & S5: Proteus penneri & CHU Tlemcen & M & $1 \mathrm{H} 30 \mathrm{~min}$ & $\mathrm{P}$ & \\
\hline \multirow[t]{2}{*}{ P6 } & P6: Pseudomonas aeruginosa & S6: Vibrio fluviatis & CHU Tlemcen & $\mathrm{F}$ & 1h $10 \mathrm{~min}$ & c & Positive \\
\hline & P'6: Staphylococcus sciuri & S'6: Staphylococcus capitis & & & & & \\
\hline \multirow[t]{2}{*}{ P7 } & P'7: Staphylococcus sciuri & S'7: Staphylococcus sciuri & CHU Tlemcen & M & $25 \mathrm{~min}$ & c & \\
\hline & P8: Vibrio fluviatis & S8: Vibrio vulgaris & CHU Tlemcen & M & 1h $15 \mathrm{~min}$ & $\mathrm{P}$ & \\
\hline P8 & P'8: Staphylococcus sciuri & $\begin{array}{l}\text { S'8: Staphylococcus } \\
\text { épidermidis }\end{array}$ & & & & & \\
\hline \multirow[t]{3}{*}{ P9 } & P9: Pseudomonas horyzihabitans & S9: Pseudomonas aeruginosa & $\begin{array}{l}\text { EH Ain } \\
\text { Temouchent }\end{array}$ & M & $20 \mathrm{~min}$ & $\mathrm{P}$ & \\
\hline & P'9: Staphylococcus simulans & $\begin{array}{l}\text { S'9: Staphylococcus } \\
\text { haemolyticus }\end{array}$ & & & & & \\
\hline & P10: Proteus penneri & S10: Proteus penneri & $\mathrm{CHU}$ Tlemcen & $\mathrm{F}$ & $1 \mathrm{~h} 10 \mathrm{~min}$ & c & \\
\hline \multirow[t]{2}{*}{ P10 } & $\begin{array}{l}\text { P"10: Chrysobacterium } \\
\text { indolegenes }\end{array}$ & S'10: Staphylococcus warneri & & & & & \\
\hline & P'10: Staphylococcus hominis & & & & & & \\
\hline \multirow[t]{2}{*}{ P11 } & P11 : Pasteurella pneumotropica & S11: Ochrabactrum antropi & CHU Tlemcen & M & 1h $15 \mathrm{~min}$ & c & \\
\hline & P'11: Staphylococcus lugdunensis & $\begin{array}{l}\text { S'11: Staphylococcus } \\
\text { épidermidis }\end{array}$ & & & & & \\
\hline \multirow[t]{2}{*}{ P12 } & P12: Ewingella americana & S12: Ewingella americana & CHU Tlemcen & M & $1 \mathrm{~h}$ & $P$ & \\
\hline & P'12: Staphylococcus epidermidis & $\begin{array}{l}\text { S'12: Staphylococcus } \\
\text { epidermidis }\end{array}$ & & & & & \\
\hline \multirow[t]{2}{*}{ P13 } & P13: Pseudomonas aeruginosa & S13: Vibrio fluviatis & CHU Tlemcen & M & $20 \mathrm{~min}$ & c & Positive \\
\hline & P'13: Staphylococcus hominis & & & & & & \\
\hline P14 & P14: Photobacterium damselae & S14: Photobacterium damselae & CHU Tlemcen & M & $25 \mathrm{~min}$ & c & \\
\hline \multirow[t]{2}{*}{ P15 } & P15: Serratia liquefaciens & S15: Enterobacter aerogenes & Kara Clinic & M & $1 \mathrm{~h}$ & c & Positive \\
\hline & & $\begin{array}{l}\text { S'15: Staphylococcus } \\
\text { épidermidis }\end{array}$ & & & & & \\
\hline \multirow[t]{3}{*}{ P16 } & P16: Serratia plymuthyca & S16: Photobacterium damselae & CHU Tlemcen & $\mathrm{F}$ & $55 \mathrm{~min}$ & $\mathrm{P}$ & Positive \\
\hline & P"16: Pasteurella pneumotropica & S'16: Staphylococcus hominis & & & & & \\
\hline & P'16: Staphylococcus hominis & & & & & & \\
\hline \multirow[t]{2}{*}{ P17 } & P17: Photobaterium damselae & S17: Moraxella & CHU Tlemcen & $\mathrm{H}$ & $1 \mathrm{~h} 15 \mathrm{~min}$ & $\mathrm{P}$ & \\
\hline & P'17: Staphylococcus épidermidis & $\begin{array}{l}\text { S'17: Staphylococcus } \\
\text { épidermidis }\end{array}$ & & & & & \\
\hline
\end{tabular}

All 17 ICDs (pockets and leads) were positive for bacteria on culture plates, and 54 bacteria (26 Gram positives and 26 Gramnegatives) were isolated and identified, with the most frequent being coagulase-negative staphylococci (CoNS) representing $46.3 \%$ (25
/54) of the total isolates (Table 2 and 3). A total of 27 bacterial isolates were recovered from the ICD pockets and 27 from the ICD leads. The most frequently isolated enteric bacteria were Pseudomonas spp, Klebsiella spp, Proteus spp., Serratia spp, and Entero- 
bacter spp identified in 9 ICDs comprising 29 out of the $54(53.7 \%)$ strains.

Other infrequently isolated bacteria from some ICDs included Ochrabactrum antropi, Ewingella americana, and Photobacterium damselae which were not associated with infectious endocarditis in the patients. Polymicrobial infection was recorded in 15 of the 17 patients (88.2\%) with varying combinations of Pseudomonas aeruginosa, Staphylococcus sciuri, Vibrio fluviatis and Staphylococcus capitis while the infection was monomicrobial in a patient $\left(\mathrm{P}_{4}\right)$ where the ICD pocket and the lead were colonized by the same Staphylococcus epidermidis strain and another patient $\left(P_{2}\right)$ where only the pocket was colonized by Staphylococcus xylosus (Table 1).

Table 2: Distribution of Gram-negative bacteria strains according to the implantable cardiac device (ICD)

\begin{tabular}{|c|c|c|c|c|}
\hline Strains & Production of slime & Tissue culture plate & MATH & Antibiotype \\
\hline S1 Moraxella & Non- Producer & Strong & & $n a^{R} a m p^{R}, a m c^{R} c r o^{R} c t x^{R} k f^{R}$ \\
\hline P3 Pseudomonas aeruginosa & Non- Producer & Strong & Strong & $\operatorname{caz}^{R} \operatorname{ctx}^{R}, \operatorname{tcc}^{R} \mathrm{cn}^{I}$ \\
\hline S3 Enterobacter cloacae & Producer & Strong & Strong & $c n^{R} c^{R} a k^{1} f^{1} c r o^{R} t e^{R} a m c^{R}$ tob ${ }^{R} a^{R} c t x^{R} a m p^{R}$ \\
\hline S3p Pseudomonas aeruginosa & Producer & Strong & Strong & $\operatorname{tob}^{I} n a^{R} c^{R} t^{R} a m c^{R} c o^{R} k f^{R} a m p^{R} c t x^{R} c z^{R}$ \\
\hline S5 Proteus penneri & Producer & Strong & & $a m c^{R} a m p^{R} k^{R} i p m^{R} c t x^{R} c r o^{R} o f x^{R} c^{2} p^{R} n a^{R} c n^{R} \operatorname{tob}^{R} \operatorname{te}^{R}$ \\
\hline P6 Pseudomonas aeruginosa & Producer & Strong & Weak & tob $^{R} a k^{I} n a^{R} c n^{R} t^{R} a m c^{R} c r o^{R} k f^{R} a m p^{R} c x^{R} c a z^{R}$ \\
\hline S6 Vibrio fluviatis & Producer & Strong & & $n a^{R} \operatorname{te}^{\mathrm{I}} a m c^{R} \mathrm{cro}^{\mathrm{I}} \mathrm{kf}^{\mathrm{R}} a m p^{R} c t x^{R}$ \\
\hline P8 Vibrio fluviatis & Producer & Strong & & $n a^{R} a m c^{R} k f^{R} a m p^{R} c t x^{R}$ \\
\hline S8 Vibrio vulgaris & Producer & Strong & & $a k^{R} n a^{R} c n^{R} t e^{R} a m c^{R} c r o^{R} k f^{R} a m p^{R} c t x^{R} c z^{R}$ \\
\hline P9 Pseudomonas horyzihabitans & Producer & Strong & Weak & tob $^{R} a k^{R} n a^{R} c n^{R} t^{R} a m c^{R} c r o^{R} k f^{R} a m p^{R} c x^{R}$ \\
\hline S9 Pseudomonas aeruginosa & Producer & Strong & Moderate & $\operatorname{cip}^{I}$ of $x^{R} \operatorname{tob}^{R} a k^{R} n a^{R} c n^{R} t^{R} a m c^{R} c r o^{R} k f^{R} a m p^{R} c t x^{R}$ \\
\hline P10 Proteus penneri & Producer & Strong & & $\operatorname{cip}^{R}$ of $x^{R} n a^{R} c t x^{I}$ \\
\hline $\mathrm{P}^{\prime \prime} 10$ Chrysobacterium indolegenes & Producer & Strong & & $\operatorname{cip}^{R}$ of $x^{R} \operatorname{tob}^{1} n a^{R} c n^{I}$ te $^{R} a m c^{I} k f^{R} c t x^{R}$ \\
\hline S10 Proteus penneri & Producer & Strong & & $n a^{1} a m c^{R} c r o^{R} k f^{R} a m p^{R} c t x^{R}$ \\
\hline P11 Pasteurella pneumotropica & Producer & Strong & & $n a^{R} a m c^{R} k f^{R} a m p^{R} c t x^{R} c r o^{R}$ \\
\hline S11 Ochrabactrum antropi & Producer & Strong & Weak & $n a^{R} a m c^{R} k f^{R} a m p^{R} c t x^{R} i p m^{R}$ \\
\hline P12 Ewingella americana & Producer & Strong & Strong & $\mathrm{ctx}^{\mathrm{R}} \mathrm{cro}^{\mathrm{R}} \mathrm{amp}^{\mathrm{R}} \mathrm{fa}^{\mathrm{I}} \mathrm{amc}^{\mathrm{R}}$ tob $^{R}$ \\
\hline S12 Ewingella Americana & Producer & Strong & Strong & $\operatorname{ctx}^{R} \mathrm{cro}^{R} a m p^{R}$ fal $^{1} a m c^{R}$ tob \\
\hline P13 Pseudomonas aeruginosa & Producer & Strong & Strong & $c t x^{R} c r o^{R} a m p^{R} c^{R} f a^{R} c a z^{R} f f^{1} t e^{R} a m c^{R} t_{0} b^{I}$ \\
\hline S13 Vibrio fluviatis & Non- Producer & Strong & & 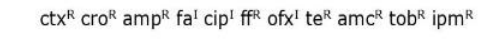 \\
\hline P14 Photobacterium damselae & Producer & Strong & Weak & $c t x^{R} c r o^{R} a m p^{R} t^{R} a m c^{R}$ \\
\hline S14 Photobacterium damselae & Producer & Strong & Weak & $c t x^{R} c r o^{R} a m p^{R} t e^{R} a m c^{R}$ \\
\hline P15 Serratia liquefaciens & Producer & Strong & Strong & $c t x^{R} c r o^{R} a m p^{R} f a^{R} f f^{1} t e^{R} a m c^{R}$ tob ${ }^{R}$ \\
\hline S15 Enterobacter aerogenes & Producer & Strong & Strong & $c t x^{R} c r o^{R} a m p^{R} c n^{R} k f^{R} t^{R} a m c^{R} \operatorname{tob}^{R} c a z^{R}$ \\
\hline P16 Serratia plymuthyca & Non- Producer & Strong & Strong & $a m c^{I} c r o^{R} k f^{R} a m p^{R} c t x^{R}$ \\
\hline S16 Photobacterium damselae & Producer & Strong & Weak & $\mathrm{cn}^{1} \operatorname{tob}^{\mathrm{I}} a m c^{R} \mathrm{cro}^{R} a m p^{R} \mathrm{ctx}^{R}$ \\
\hline P17 Photobaterium damselae & Producer & Strong & Weak & $\operatorname{tob}^{\mathrm{I}} \mathrm{cro}^{\mathrm{T}}$ \\
\hline S17 Moraxella & Non- Producer & Strong & Strong & $\mathrm{ctx}^{R} \mathrm{cro}^{R} \mathrm{amp}^{R} \mathrm{te}^{\mathrm{l}} \mathrm{amc} \mathrm{c}^{R}$ \\
\hline P"16 Pasteurella pneumotropica & Non- Producer & Weak & & $c n^{1} \operatorname{tob}^{1} a m c^{R} c r o^{R} k f f^{R} a m p^{R} c t x^{R}$ \\
\hline
\end{tabular}


Table 3: Distribution of Gram-positive bacteria strains according to the implantable cardiac device (ICD)

\begin{tabular}{|c|c|c|c|c|}
\hline Strains & Production of slime & Tissue culture plate & Math & Antibiotype \\
\hline P'2 Staphylococcus xylosus & Non- Producer & Strong & & $n a^{I} k^{R} v a^{I}$ \\
\hline P'9 Staphylococcus simulans & Producer & Strong & Strong & $n a^{R} v a^{T} t e^{R}$ \\
\hline $\mathrm{P} " 10$ Chrysobacterium indolegenes & Producer & Strong & & $c p^{R}$ of $x^{R}$ tob $b^{I} a^{R} c n^{I}$ te temc $^{I} k^{R} c t x^{R}$ \\
\hline S'1 Staphylococcus xylosus & Producer & Moderate & Weak & $n a^{1} k^{R} v a^{1}$ \\
\hline P'4 Staphylococcus épidermidis & Non- Producer & Moderate & Strong & $o x^{R}$ tob ${ }^{R} n^{R} v a^{I} o x^{R}$ \\
\hline S'4 Staphylococcus épidermidis & Non- Producer & Moderate & & $o x^{R} r^{S} t_{0 b}^{R} c n^{R} v a^{1} o x^{R}$ \\
\hline P'5 Staphylococcus sciuri & Producer & Moderate & Strong; Hydrophobe & $n a^{R} a m c^{R} c r o^{I} a m p^{R} c t x^{R} k f^{R} v a^{I}$ \\
\hline S'6 Staphylococcus capitis & Producer & Moderate & Moderate & $n a^{R} a m c^{R} c r 0^{I} a m p^{R} c t x^{R} k f^{R} v a^{l}$ \\
\hline P'7 Staphylococcus sciuri & Producer & Moderate & Weak & $n a^{I} c^{I} v a^{I}$ te $^{R}$ \\
\hline S'7 Staphylococcus sciuri & Producer & Moderate & Weak & $n a^{1} c^{1} v a^{1} t e^{R}$ \\
\hline S'8 Staphylococcus épidermidis & Producer & Moderate & Weak & $o x^{I} n^{R} c^{R} v^{I}$ te $e^{R}$ tob \\
\hline P'10 Staphylococcus hominis & Producer & Moderate & Strong; Hydrophobe & $\mathrm{cro}^{\mathrm{I}} \mathrm{kf}^{\mathrm{f}} \mathrm{ctx}^{\mathrm{R}}$ \\
\hline S'10 Staphylococcus warneri & Producer & Moderate & Weak & $n a^{I} a m c^{R} c^{I} k f^{R} a m p^{R} c x^{R} f f r^{I}$ \\
\hline P'11 Staphylococcus lugdunensis & Producer & Moderate & Weak & $n a^{I} k f^{I} a m p^{I} c t x^{R}$ \\
\hline S'11 Staphylococcus épidermidis & Producer & Moderate & Strong & $n a^{I} a m c^{R} c r 0^{R} k f^{R} a m p^{R} c t x^{R}$ \\
\hline P'12 Staphylococcus epidermidis & Producer & Moderate & Weak & $o x^{R} n a^{R} n^{I} v^{I} t^{R}$ tob \\
\hline P'13 Staphylococcus hominis & Non- Producer & Moderate & Weak & $\mathrm{va}^{\mathrm{l}} \mathrm{te}^{\mathrm{R}}$ \\
\hline S'15 Staphylococcus épidermidis & Producer & Moderate & Weak & $o x^{R} n a^{S} p n v^{R} n^{S} c^{S} k^{S} c p^{S} p^{f} v^{S} v a^{I} t^{R} r a^{R} t^{2} b^{R}$ \\
\hline P'16 Staphylococcus hominis & Producer & Moderate & Weak & $n a^{l} v^{I}$ \\
\hline S'16 Staphylococcus hominis & Producer & Moderate & & $o x^{I} n a^{R} n^{1} v a^{1} t e^{R}$ \\
\hline P'17 Staphylococcus warneri & Producer & Moderate & Strong & $n a^{1} v a^{1}$ \\
\hline P'6 Staphylococcus sciuri & Producer & Weak & Weak & $n a^{R} v a^{I}$ \\
\hline P'8 Staphylococcus sciuri & Producer & Weak & Weak & $\operatorname{tob}^{1} a k^{1} n a^{R} c n^{R} t^{R} a m c^{R} c r 0^{R} k f^{R} a m p^{R} c x^{R}$ \\
\hline S'9 Staphylococcus haemolyticus & Producer & Weak & Strong; Hydrophobe & $n a^{R} a m c^{R} c r o^{R} k^{f R} a m p^{R} c t x^{R}$ \\
\hline $\mathrm{S}^{\prime} 12$ Staphylococcus chromogenes & Producer & Weak & Weak & $n a^{I} k^{R} v a^{I}$ te ${ }^{I} t o b^{R}$ \\
\hline S'17 Staphylococcus épidermidis & Producer & Weak & Weak & $n a^{I} k^{R} v a^{I}$ tob ${ }^{R}$ \\
\hline
\end{tabular}


This study of antibiotic susceptibility of the isolated strains in the four hospital centers showed resistance at a significant level against several antibiotics (Fig 1 and Fig 2). Fig 1 is a histogram illustrating the resis tant pattern of the Gram-negative bacterial isolates showing high resistance rate $(>90 \%)$ to amoxicillin, clavulanic acid, cefotaxime, and gentamycin, with 3 isolates resistant to imipenem. None of the staphylococcal strains was resistant to vancomycin. Fig 2 is a histogram illustrating the resistance pattern of the Gram-positive isolates.

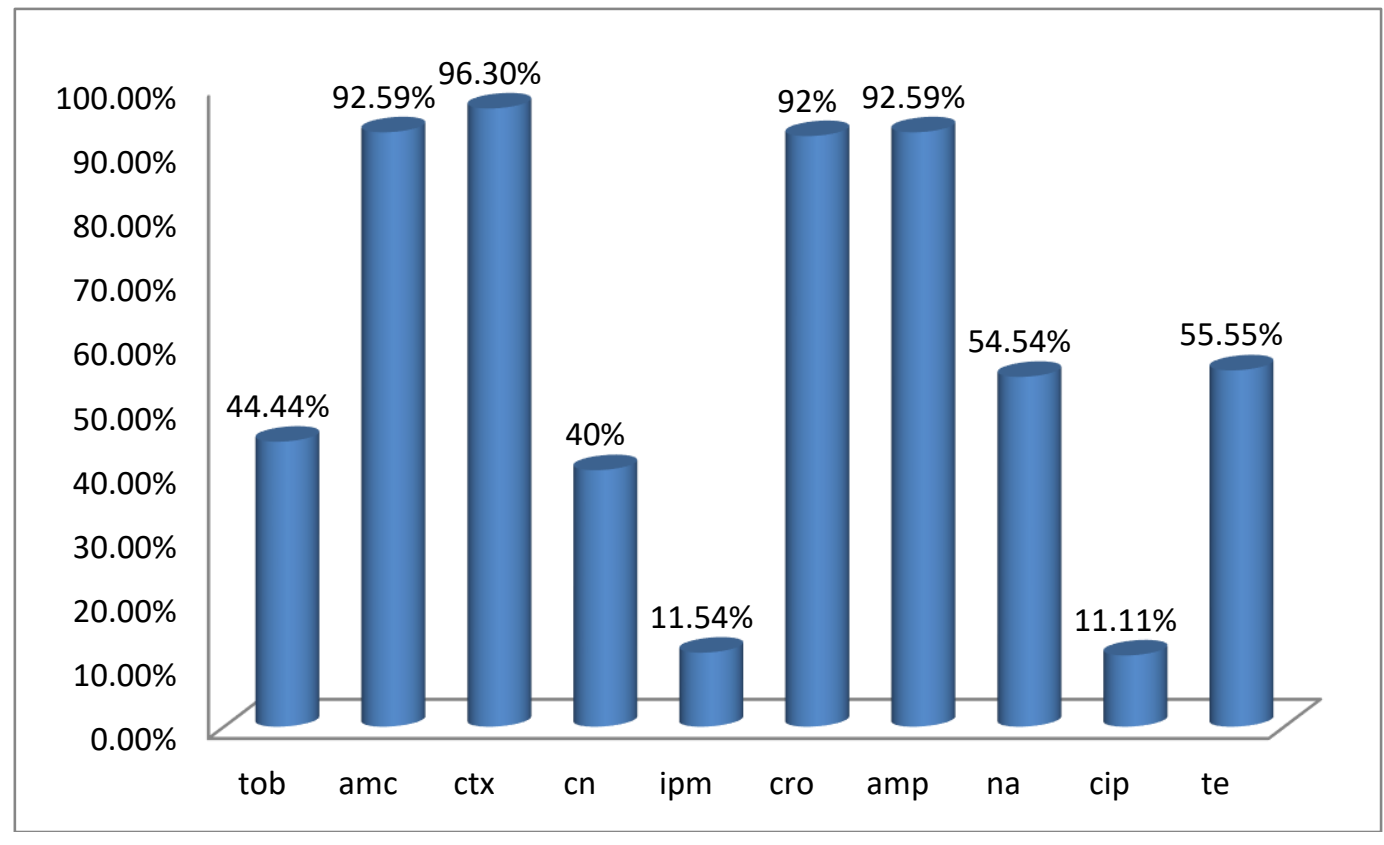

Fig 1: (Histogram $\mathrm{A}_{1}$ ): Resistance rate of Gram-negative bacteria isolates

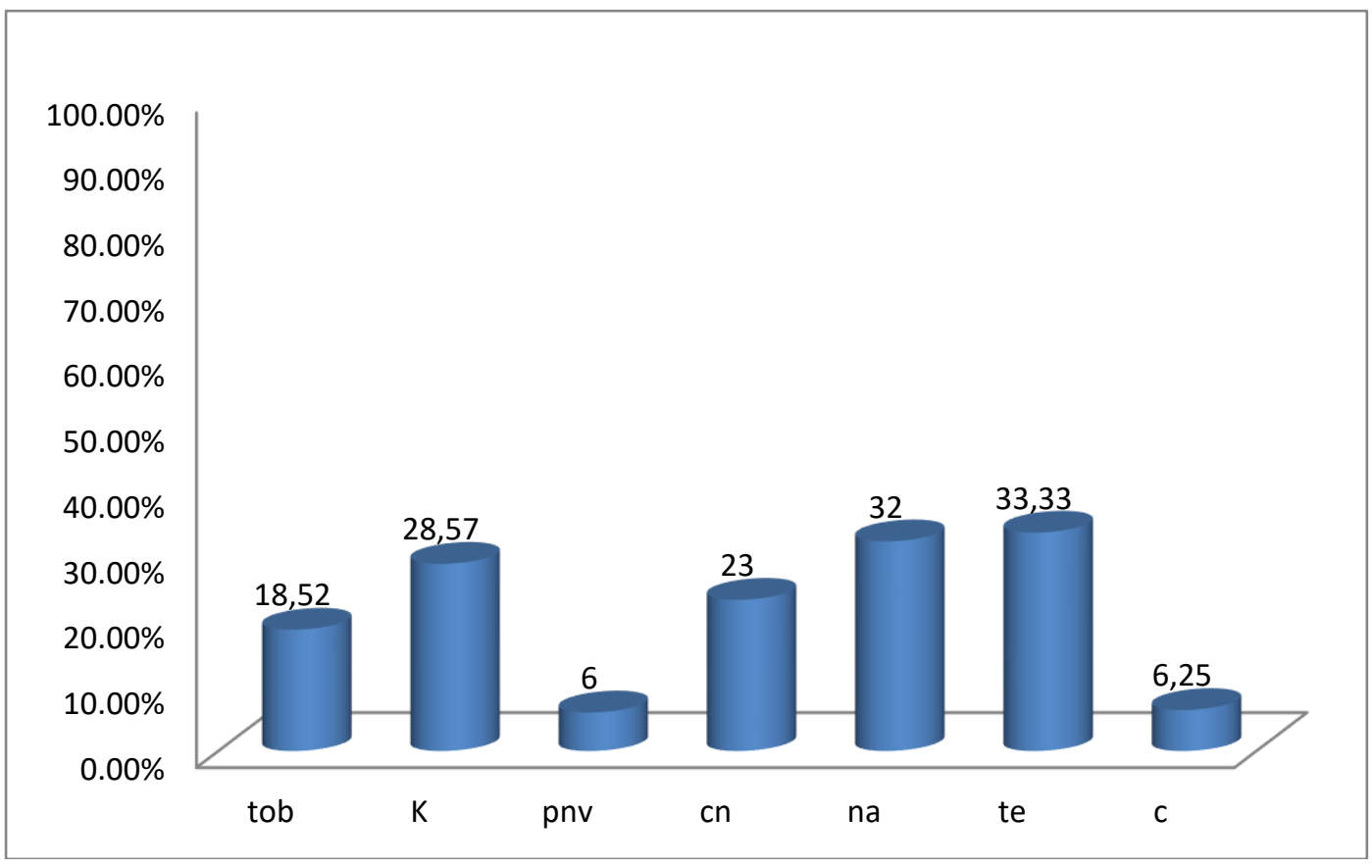

Fig 2: (Histogram $\mathrm{A}_{2}$ ): Resistance rate of Gram-positive bacteria isolates 


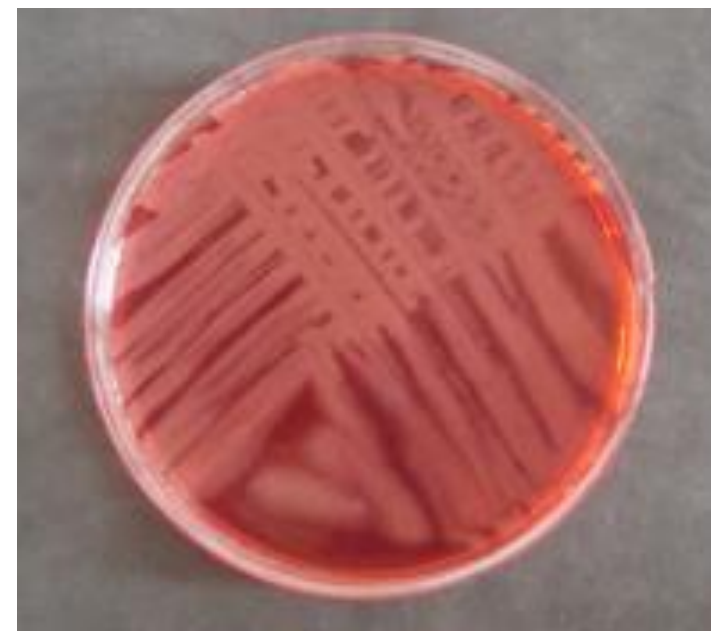

A

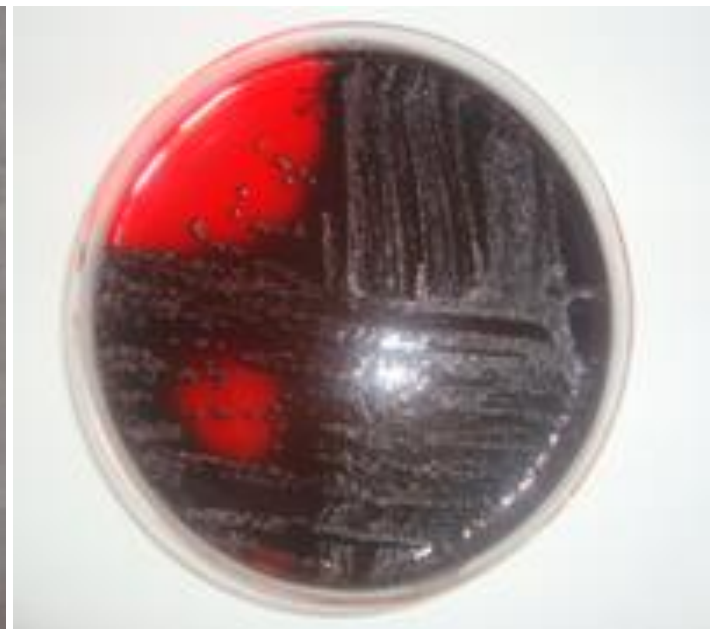

B

Fig 3: Slime production in the strains isolated on Congo red medium

A: slime generating, $\mathrm{B}$ : non-slime generating

\section{Results of biofilm detection}

The results of the biofilm production by the bacterial isolates with the qualitative CRA and the quantitative TCP methods are presented in Table 2. Out of the 54 bacteria isolates, $44(81.5 \%)$ were slime producers by the CRA method and 24 of them were strong (very good) biofilm producers using the TCP method, with OD $570>0.240$. The TCP method detected biofilms in 48 (88.9\%) of 54 isolates with different intensities; 30 (55\%) isolates were strong producers, 18 (33.3\%) isolates were moderate, $6(11.1 \%)$ isolates were low biofilm producers, while 10 (18.5\%) were non-producers of biofilms. Thirty-seven $(68.5 \%)$ of the 54 isolates were positive by both TCP and RCA methods. Fig 3 is a photograph of CRA plate showing slime prod- uction by a positive isolate $(A)$ and non-slime production by a negative isolate $(B)$.

\section{Result of hydrophobicity}

The results of the microbial adhesion to the solvent (hydrophobicity) are summarized in Figs 4 and 5. A total of 14 Gram positive and 7 Gram negative bacteria isolates had affinity to hexadecane (apolar solvent) suggesting a weak hydrophilic character. On the other hand, 17 (41.5\%) isolates were relatively hydrophobic between $1 \%$ and $96 \%$, and $2(5 \%)$ strains were moderately hydrophobic. Fig 4 is a histogram of the cell surface hydrophobicity of the Gram-negative bacteria isolates and Fig 5 is a histogram of the cell surface hydrophobicity of Gram-positive bacteria isolates.

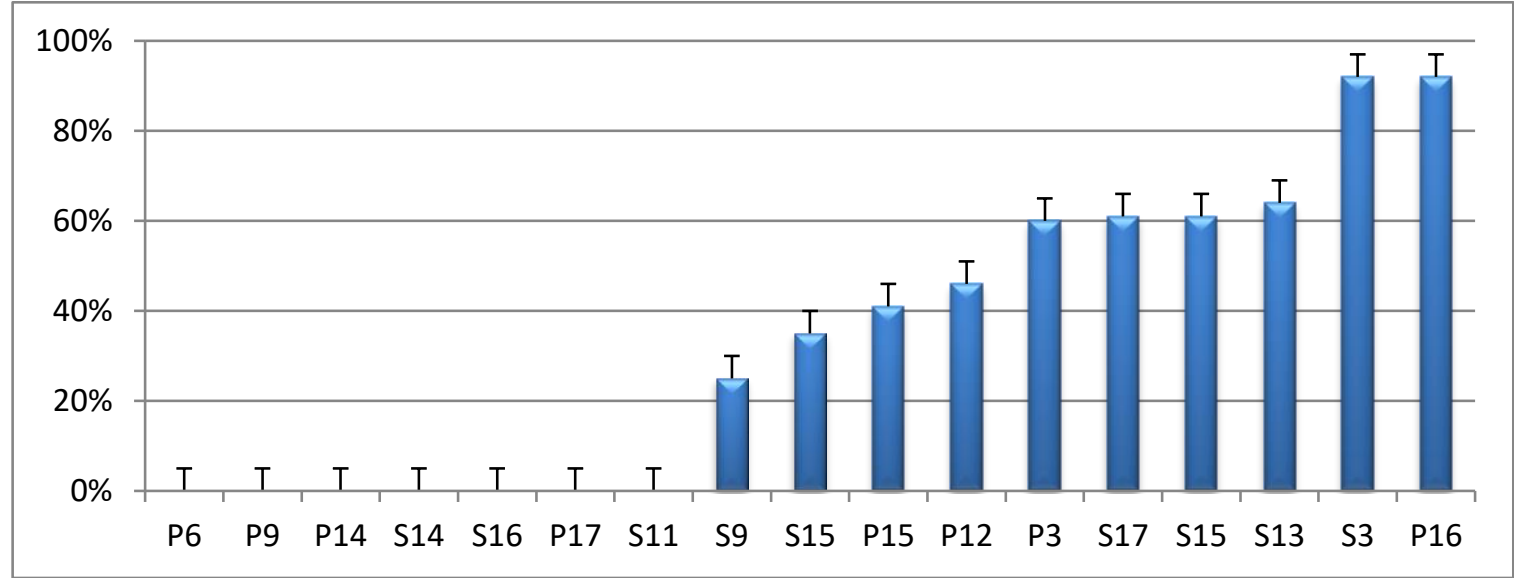

Fig 4: (Histogram $B_{1}$ ): Cell surface hydrophobicity of Gram-negative bacteria isolates 


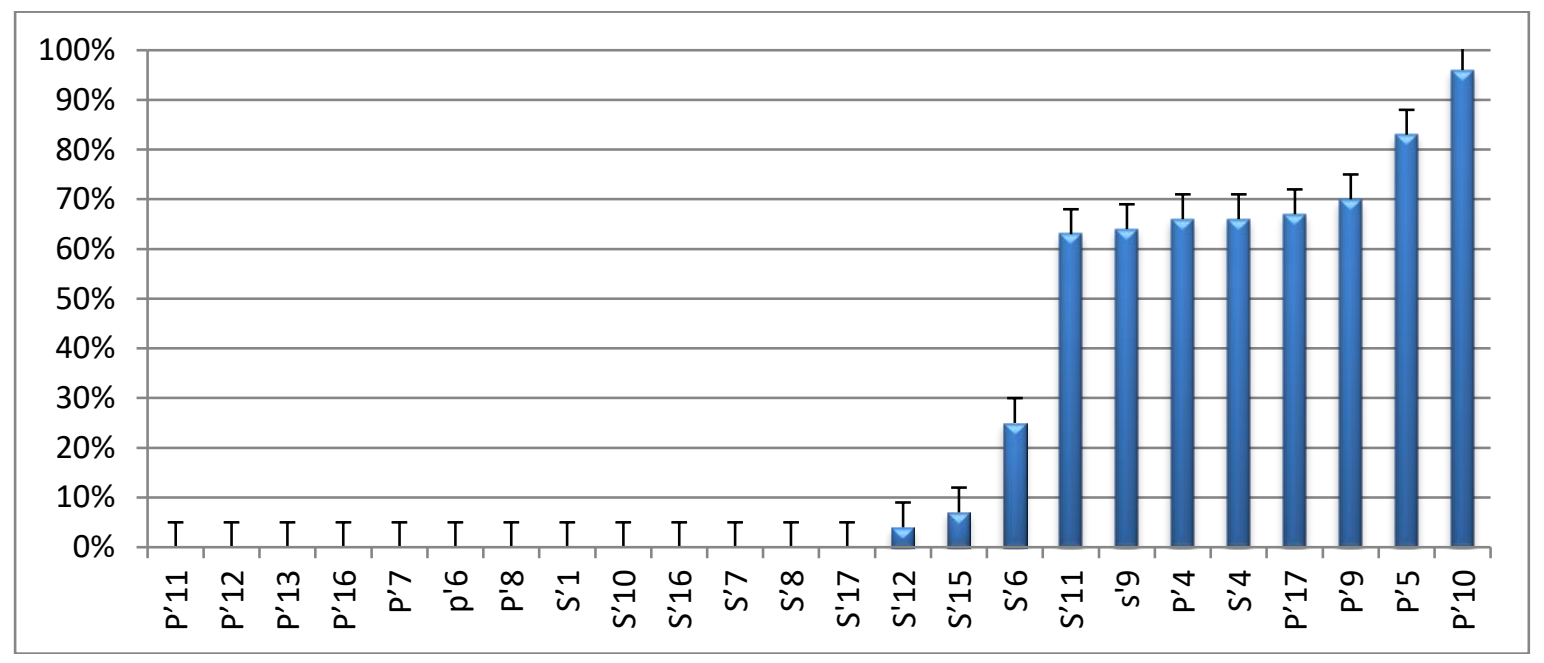

Fig 5: (Histogram B2): Cell surface hydrophobicity of Gram-positive bacteria isolates

\section{Discussion:}

ICDs have become increasingly of great importance in the management of heart disease in many countries around the world with a great impact on the quality of life of patients (10). The advances in the development of permanent pacemakers and the technologies of implantable cardiac defibrillators have helped this process. Nevertheless, with the increase in ICD implantations, infections on these devices have correspondingly increased. To the best of our knowledge, this is the first and only study on the microbiological characteristics of ICD infections in Algeria. Although our data reflect the epidemiology of ICD infections in the region, they could be of great interest and provide useful information for ICD infections management in other regions. In recent decades, the rate of these ICD infections has increased worldwide from $0.13 \%$ to $19.9 \%$ with an average rate of $10 \%$ $(15,26)$. The overall incidence of infection on ICD implantable cardiac devices is estimated at 1.9 per thousand per year (27). According to an estimate by Camus et al., there are more than three million patients on pacemaker (PM) worldwide and 180,000 carriers of implantable defibrillator (ID) (28). Among the complications occurring after the implantation of an ICD, infection is certainly the most serious (29). In this retrospective study of 21 months, of a total of 315 ICD implantations in 4 hospitals, 17 patients presented with infection of their ICDs, giving an incidence rate of $5.4 \%$. All the infections were diagnosed and confirmed by specialist cardiologists with clinical presentations that included erythema, heat, redness with or without purulent drainage, sepsis and infectious endocarditis. This rate is quite high when compared to other studies such as the French study by Klug and his team (30) in 2000 where the incidence of infectious complications was only $0.68 \%$. In 2010 , Baddour et al., (31) and Voigt et al., (32) estimated the incidence to be between $0.13 \%$ and $20 \%$, which was related to the increase in the implantation rate of ICDs in the world in general. Of the 17 infected patients, 16 had infection of their pacemakers (PM), giving an incidence rate of $5.1 \%$ in the study. In the literature, the incidence of pacemaker infections is known to vary between $0.13 \%$ and $19.9 \%$ occurring mostly at the site of implantation of the generator, and in $10 \%$ of cases, it is the cause of infectious endocarditis (33). Only one patient had an infection after defibrillator implantation (DI) representing an incidence of $0.3 \%$, which is known to vary between $0.7 \%$ and $1.2 \%$ (34).

The infection of an ICD pocket is a rare but much feared early complication, which is defined as an infection occurring within the six months following the procedures (35) and often accompanied by fever and local signs that require urgent antibiotic therapy and immediate removal of the device (36). In our study, 6 of the 17 $(35.3 \%)$ infected patients had early infection of ICD. According to the study by Ben Abid et al., (37), $25 \%$ of ICD infections are early and $42 \%$ are late, with no significant difference reported with respect to the type of infectious organisms. Nine of the 17 (52.9\%) infected patients had an outward displacement of the pocket, a rate that is considerably higher 
compared to the study of Klug et al., (38) where $29.5 \%$ of the patients presented with an outward displacement of the pocket in a study of 105 patients.

There are various factors predisposing to ICD infection that have been reported including advanced age, diabetes, cancer and immunosuppression, anticoagulation, presence of a temporary stimulation lead, duration of intervention, and the surgeon's experience (39). Indeed, diabetes mellitus and age are predisposing factors in most of our patients. The highest rate of infection was observed in the age group 60 to 75 years who were mainly male patients among whom $29.4 \%$ were diabetic. A total of 15 patients had positive cultures on the lead and the pocket of their ICDs, 8 of whom were infected by the same bacterium. Victor et al., (40) reported that the infection of the lead is associated with that of the pocket in every two cases. This infection can occur in isolation and in one fourth of cases a few weeks after implantation (40). The culture of the removed devices allows the possibility of an etiological diagnosis of ICD infection in the vast majority of cases. This means that microbiological analysis is mandatory in the case of suspicion of an ICD infection.

A total of 54 bacteria were isolated and identified from the ICDs; $46.3 \%$ Gram positive bacteria were isolated, which were mostly CoNS with predominance of $S$. epidermidis. These findings closely agree with to those reported by Gill et al., (41) who estimated that about $41.2 \%$ of ICD infections were caused by CoNS. The dominant role of staphylococci is consistent with what has been previously reported where staphylococcal species accounted for 60 to $80 \%$ of the cases in most of the reported studies $(36,42,44-44)$. In several studies, $S$. epidermidis was the most frequently isolated CoNS species and constitutes a significant part of the normal bacterial flora of human skin and mucous membranes from where it is easily introduced as a contaminant during surgical implantation of the polymer device (45).

Gram-negative bacteria constituted $53.7 \%$ of the isolated bacteria, with high frequency of enteric bacteria such as Proteus spp, Serratia spp, Enterobacter spp and Pseudomonas spp. These bacteria which are involved contamination and care-related infections were recovered twice in the space of a few weeks in two different hospitals. This implies either contamination or poor technique during the surgical procedure. We noted the exceptional recovery of three uncommon bacteria species; Ewingella ameri- cana, Ochrabactrum antropi, and Photobacterium damselae from the ICDs in our study, which have been reported only by a few authors such as Pien et al., (46), Mahmood et al., (47), and Austin et al., (48). Nevertheless, the important causative microorganisms of ICD infections are CoNS followed by Gram-negative bacilli, fungi, nontuberculous mycobacteria and Bulkholderia cepacia. The bacterial complex is a rare cause of ICD infection with only a few cases of ICD pocket infection reported in the literature (41,49,50-51). ICD infections are mainly caused by the contamination of the local bacterial flora during implantation. Skin micro-organisms migrate from the insertion site along surface of the lead, colonize the intravascular distal portion, and finally infect the blood (52).

Nearly all of the isolated Gramnegative bacteria in this study were resistant to ampicillin, amoxicillin, cefotaxime, and ceftriaxone. Although imipenem was highly active on the tested strains, three isolates were resistant to this antibiotic. These strains were also resistant to quinolones with rates ranging from $11.1 \%$ for ciprofloxacin to $54.5 \%$ for nalidixic acid. Resistance rates of isolates to tetracycline and tobramycin were $55.5 \%$ and $44.4 \%$ respectively. Unlike Gramnegative bacteria, CoNS isolates displayed a higher level of sensitivity but with some were resistant to nalidixic acid and tetracycline. According to 2008 Camus study (28), majority of staphylococci are multi-sensitive with especial sensitivity to aminoglycosides, but the isolates in our study were resistant to kanamycin $(28.5 \%)$ and tobramycin (18.5\%).

The development and persistence of bacterial infections are often associated with the foreign equipment and materials used in the devices and the ability of these bacteria to adhere to them. The bacterial strains implanted on the surface of a device are inserted and protected by a dense polysaccharide extracellular matrix and are more resistant to antibiotics, constituting the biofilm that can be detected by quantitative and qualitative techniques (1). Adherence and persistence of CoNS infection are often associated with foreign materials. Adhesion is primarily related to non-specific physicochemical forces, and then to specific interaction of the bacterial surface adhesins (not directly with the device) with host proteins coating the device (53). CoNS may adhere directly to the device plastic polymers via fimbria-like surface protein structures or via a capsular polysaccharidic adhesin (54). Bacteria may also adhere to host matrix proteins such as fibrinogen, fibronectin, and 
collagen that coat the surface of an implanted device (55). The layers of bacteria on the surface of an implanted device are encased in an extracellular slime made of a polysaccharide intercellular adhesion and constitute the biofilm $(56,57)$. Microbes in a biofilm are protected by this dense extracellular matrix and are more resistant to antibiotics and host defenses. Biofilm forming bacteria are therefore a public health challenge for those requiring indwelling medical devices (56). In our study, the presence of infection was influenced by the ability of 48 out of 54 strains to form a biofilm, whatever the support (pocket and leads), and thus representing a significant risk factor. The TCP technique, which is the most widely used method, is considered the 'gold standard' test for sensitive, accurate and reproducible screening method for the detection of biofilm production in clinical isolates (58). The adherence capacity of clinical isolates to host cells depends on bacterial surface properties such as hydrophobicity. In a recent study, it was reported that there is a significant difference in cell surface load between group B streptococci treated with antibiotics and those untreated (59). The determination of the acid-base properties (acceptor electron, donor electron) may be of great importance in many research areas of phagocytosis and microbial adhesion (60).

Our data indicated that affinity to hexadecane (apolar solvent) suggests low hydrophilicity for the majority of the bacteria isolated from surfaces of hydrophobic medical device. This contradicts several studies which reported that hydrophobic cells tend to adhere to hydrophobic substrates, while hydrophilic cells tend to adhere to hydrophilic substrates $(61,62)$. It has also been suggested that there is a positive correlation between the degree of bacterial hydrophobicity and adhesion to abiotic surfaces (63). Our finding can be explained by the fact that these bacteria adhere to host proteins such as fibrinogen, fibronectin, and collagen which cover the surface of the implanted device, making it hydrophobic (55). The comparison between the biofilm formation (by RCA and TCP), hydrophobicity and antimicrobial resistance of the 54 bacteria isolates revealed that there is no significant association in terms of isolates but that there is correlation between biofilm formation and resistance to antibiotics, with the strong biofilm producers resistant to more than one antibiotic.

\section{Conclusion:}

This study is the first in Algeria to describe microbiological characteristics of ICD infections. CoNS were the most frequent cause of ICD infections in our study but Pseudomonas aeruginosa and Serratia spp were also significant cause of bacteremia in patients with ICD, with comparable rate to the CoNS. This situation may imply bacteria contamination or poor technique during surgical procedure for implantation of the ICDs.

The bacteria in the biofilm were protected, more resistant and tolerated high concentrations of antibiotics, and thus played a major role in the development of ICD infections. Despite the improvements in ICD design and implantation techniques, ICD infection remains a serious challenge. It is therefore essential that proper infection prevention and control practices be put in place as well as strict indications for antibiotic prophylaxis during implantation of ICDs.

\section{Conflict of interest statement:}

No conflict of interest is declared.

\section{Acknowledgements:}

The authors acknowledge with special thanks, the medical and paramedical staff of CHU Tlemcen Algeria and Dr Benahmed Khaled, cardiologist of the $\mathrm{EH}$ ain Temouchent Algeria, for his collaboration.

\section{References:}

1. Bongiorni, M. G., Tascini, C., Tagliaferri, E., et al. Microbiology of cardiac implantable electronic device infections, Europace. 2012; 14: 1334 1339. doi:10.1093/europace/eus044.

2. Bluhm, G. L. Pacemaker infections. A 2-year follow-up of antibiotic prophylaxis., Scand J Thorac Cardiovasc Surg. 1985; 19: 231235.

3. Kearney, R. A., Eisen, H.J., and Wolf, J. E. Nonvalvular infections of the cardiovascular system. Ann Intern Med. 1994; 121 (3): 219230.doi:10.7326/0003-4819-121-3-19940801000010.

4. Tascini, C., Bongiorni, M. G., Gemignani, G., et al. Management of Cardiac Device Infections: A Retrospective Survey of a Non-Surgical Approach Combining Antibiotic Therapy with Transvenous Removal. J Chemother. 2006; 18 (2): 157-163. doi:10.1179/joc.2006.18.2.157.

5. Baddour, L. M., Epstein, A. E., Erickson, C. C., et al. Update on cardiovascular implantable electronic device infections and their management: a scientific statement from the 
American Heart Association, Circulation. 2010; 121(3):458-477.doi:10.1161/circulationaha.109.192665.

6. Dababneh, A. S., and Sohail, M. R. Cardiovascular implantable electronic device infection A stepwise approach to diagnosis and management. Clevel Clin J Med. 2011; 78 (8): 529537. doi:10.3949/ccjm.78a.10169.

7. Santos, A. P. A., Watanabe, E.. and de Andrade, D. Biofilm on artificial pacemaker: fiction or reality?, Arq Bras Cardiol. 2011; 97: e113120. doi:10.1590/S0066-782X2011001400018.

8. Sohail, M. R. Hussain, S., Le, K. Y., et al. Risk factors associated with early- versus late-onset implantable cardioverter-defibrillator infections., J Interv Card Electrophysiol. 2011; 31(2): 171183. doi:10.1007/s10840-010-9537-x.

9. Nagpal, A., Baddour, L. M. and Sohail, M. R. Microbiology and pathogenesis of cardiovascular implantable electronic device infections. Circ Arrhythmia Electrophysiol. 2012; 5: 433-441. doi:10.1161/CIRCEP.111.962753.

10. Anselmino, M., Vinci, M. Comoglio, C., et al. Bacteriology of infected extracted pacemaker and ICD leads. J Cardiovasc Med (Hagerstown). 2009; 10:693-698. doi:10.2459/JCM.0b013e32832b3585.

11. Viola, G. M. Awan, L. L., and Darouiche, R.O. Nonstaphylococcal infections of cardiac implantable electronic devices. Circulation. 2010;121(19):2085-2091. doi:10.1161/CIRCULATIONAHA.110.936708.

12. Agostinho, A., James, G., Wazni, O., Citron, M., and Wilkoff, B. D. Inhibition of Staphylococcus aureus biofilms by a novel antibacterial envelope for use with implantable cardiac devices. Clin Transl Sci. 2009; 2(3): 193-198.

13. Stoodley, P., Sauer, K., Davies, D. G., and Costerton, J. W. Biofilms as Complex Differentiated Communities. Annu Rev Microbiol. 2002;56:187-209.

doi:10.1146/annurev.micro.56.012302.160705

14. Khardori, N., and Yassien, M. Biofilms in devicerelated infections. J Ind Microbiol. 1995; 15: 141-147. doi:10.1007/BF01569817.

15. Ruttmann, E., Hangler, H. B., Kilo, J., et al. Transvenous pacemaker lead removal is safe and effective even in large vegetations: an analysis of 53 cases of pacemaker lead endocarditis. Pacing Clin Electrophysiol. 2006; 29: $231-236$.

doi: $10.1111 / j .15408159 .2006 .00328 . x$.

16. Costerton, J. W., Lewandowski, Z., Caldwell, D. E., Korber, D. R., and Lappin-Scott, H. M. Microbial biofilms. Annu Rev Microbiol. 1995;49:711-745.

doi:10.1146/annurev.mi.49.100195.003431.

17. Bonkat, G., Rieken, M., Rentsch, C. A., et al. Improved detection of microbial ureteral stent colonisation by sonication. World J Urol. 2011; 29: 133-138. doi:10.1007/s00345-010-0535-5.

18. Sampedro, M. F. Huddleston, P. M., Piper, K. E., et al. A biofilm approach to detect bacteria on removed spinal implants., Spine (Phila. Pa. 1976).2010;35:1218-1224.

doi:10.1097/BRS.0b013e3181c3b2f3.

19. Kara Terki, I., Hassaine, H. Bellifa, S., et al. Evaluation of biofilm formation of Klebsiella pneumoniae isolated from medical devices at the University Hospital of Tlemcen, Algeria. Afr J Microbiol Res. 2013; 7: 5558 - 5564. doi:10.5897/AJMR12.2331.

20. CASFM. 2010; Antibiogram Committee of the French Society of Microbiology.

21. Christensen, G. D. Simpson, W. A., Younger, J. J., et al. Adherence of coagulase-negative staphylococci to plastic tissue culture plates: a quantitative model for the adherence of staphylococci to medical devices. J Clin
Microbiol.1985;22:996-1006.

http://www.ncbi.nlm.nih.gov/pubmed/3905855.

22. Terki, I. K., Hassaine, $\mathrm{H}$., and Bellifa, S. Biofilm production and antibiotic susceptibility profiles of staphylococcus strains isolates from urinary catheter at the university hospital center of Tlemcen (Algeria). IJSRST. 2015; 1 (6): 77-85.

23. Satorres, S. E., and Alcaraz, L. E. Prevalence of icaA and icaD genes in Staphylococcus aureus and Staphylococcus epidermidis strains isolated from patients and hospital staff. Centr Eur J Publ HIth. 2007; 15 (2): 87-90.

24. Rosenberg, $M$. Bacterial adherence to polystyrene: a replica method of screening for bacterial hydrophobicity. Appl Environ Microbiol. 1984; 42: 375-377.

25. Imane, M., Hafida, H., Samia, B., Meriem, L., Ibtissem, K. T., and Ryad, D. Biofilm formation by Acinetobacter baumannii isolated from medical devices at the intensive care unit of the University Hospital of Tlemcen (Algeria). Afr J Microbiol Res. 2014; 8: 270-276. doi:10.5897/AJMR2013.6288.

26. Hall-Stoodley, L. Costerton, J. W., and Stoodley, P. Bacterial biofilms: from the natural environment to infectious diseases. Nat Rev Microbiol.2004;2:95-108.doi:10.1038/nrmicro821.

27. Habib, G. Hoen, B. Tornos, P., et al. Guidelines on the prevention, diagnosis, and treatment of infective endocarditis (new version 2009): the Task Force on the Prevention, Diagnosis, and Treatment of Infective Endocarditis of the European Society of Cardiology (ESC). Endorsed by the European Society of Clinical Microbiology and Infectious Diseases (ESCMID) and the International Society of Chemotherapy (ISC) for Infection and Cancer. Eur Heart J 2009; 30: 2369-2413. doi:10.1093/eurheartj/ehp285.

28. Camus, C. Infections graves liées aux stimulateurs cardiaques et défibrillateurs implantables. Reanimation. 2008; 17: 225 - 232 doi:10.1016/j.reaurg.2008.01.004.

29. Touboul, P. Indications du défibrillateur automatique implantable ventriculaire, 2007. https://sfcardio.fr/sites/default/files/pdf/reco0010.pdf

30. D. Klug, D., M. Balde, M., and D. Pavin, D., et al. PEOPLE Study Group, Risk factors related to infections of implanted pacemakers and cardioverter-defibrillators: results of a large prospective study. Circulation. 2007; 116: 1349-1355.

doi:10.1161/Circulationaha.106.678664

31. Baddour, L.M., Epstein, A.E., and Erickson, C.C., et al. A summary of the update on cardiovascular implantable electronic device infections and their management: a scientific statement from the American Heart Association. J Am Dent Assoc 2011; 142 (2): 159-165.doi: $10.14219 /$ jada.archive.2011.0058.

32. Voigt, A., Shalaby, A., and Saba, S. Continued rise in rates of cardiovascular implantable electronic device infections in the United States: temporal trends and causative insights. Pacing Clin Electrophysiol. 2010; 33: 414-419. doi: $10.1111 /$ j.1540-8159.2009.02569.x

33. Conklin, E. F. Giannelli, S., and Nealon, T. F. Four hundred consecutive patients with permanent transvenous pacemakers. J Thorac Cardiovascular Surg. 1975; 69: 1-7 http://www.ncbi.nlm.nih.gov/pubmed/1110570

34. Moss, A. J., Daubert, J., and Zareba, W. MADITII: clinical implications. Card Electrophysiol Rev. 2002;6:463-465.

http://www.ncbi.nlm.nih.gov/pubmed/12438829

35. Sandoe, J. A. T., Barlow, G. Chambers, J. B., et al. Guidelines for the diagnosis, prevention and management of implantable cardiac electronic device infection. Report of a joint Working Party project on behalf of the British Society for 
Antimicrobial Chemotherapy (BSAC, host organization), British Heart Rhythm Society (BHRS), British Cardiovascular Society (BCS), British Heart Valve Society (BHVS) and British Society for Echocardiography (BSE). J Antimicrob Chemother. 2015; 70: 325 - 359. doi: $10.1093 / \mathrm{jac} / \mathrm{dku} 383$.

36. del Río, A. Anguera, I., Miró, J. M., et al. Hospital Clínic Endocarditis Study Group, Surgical treatment of pacemaker and defibrillator lead endocarditis: the impact of electrode lead extraction on outcome., Chest. 2003; 124: 1451- 1459.

37. http://www.ncbi.nlm.nih.gov/pubmed/14555579 AlBishawi, A and Thapur, M. Delayed Pacemaker Generator Pocket and Lead Primary Infection Due to Burkholderia cepacia. Am J Case Rep. 2017; 18:855-858.

http://www.ncbi.nlm.nih.gov/pubmed/28769025 Klug, D., Wallet, F., Lacroix, D., et al. Local symptoms at the site of pacemaker implantation indicate latent systemic infection., Heart. 2004; 90:882-886.doi:10.1136/hrt.2003.010595.

39. Villamil Cajoto, I., Rodríguez Framil, M., Van den Eynde Collado, A., José Villacián Vicedo, M. and Canedo Romero, C. Permanent transvenous pacemaker infections: an analysis of 59 cases. Eur J Intern Med. 2007; 18:484-488.

40. Victor, F., De Place, C., Camus, C., et al. Pacemaker lead infection: echocardiographic features, management, and outcome., Heart. 1999;81:82-87.

http://www.ncbi.nlm.nih.gov/pubmed/10220550

41. Gill, J. S., Singh, N., and Khanna, S. P. Risk of cardiac pacemaker pocket infection in a tertiary care hospital. Indian J Pathol Microbiol. 2017; 60: 185-188. doi:10.4103/IJPM.IJPM_190_16.

42. Camus, C., Leport, C., Raffi, F., Michelet, C., Cartier, F., and Vilde, J. L. Sustained bacteremia in 26 patients with a permanent endocardial pacemaker: assessment of wire removal. Clin Infect Dis. 1993; 17: 46 - 55 http://www.ncbi.nlm.nih.gov/pubmed/8353245

43. Sohail, M. R., Uslan, D. Z., Khan, A. H., et al. Management and outcome of permanent pacemaker and implantable cardioverterdefibrillator infections. J Am Coll Cardiol. 2007; 49:1851-1859.doi:10.1016/j.jacc.2007.01.072.

44. Klug, D., Lacroix, D., Savoye, C., et al. Systemic infection related to endocarditis on pacemaker leads: clinical presentation and management. Circulation. 1997; 95: 2098 - 2107. http://www.ncbi.nlm.nih.gov/pubmed/9133520

45. Otto, M. Staphylococcal biofilms. Curr Top Microbiol Immunol. 2008; 322: 207-228. http://www.ncbi.nlm.nih.gov/pubmed/18453278 Pien, F. D., and Bruce, A. E. Nosocomial Ewingella americana bacteremia in an intensive care unit. Arch Intern Med. 1986; 146: 111-112 http://www.ncbi.nlm.nih.gov/pubmed/3942442

47. Mahmood, M. S., Sarwari, A. R., Khan, M. A., Sophie, Z., Khan, E., and Sami, S. Infective endocarditis and septic embolization with Ochrobactrum anthropi: case report and review of literature. J Infect 2000; 40: 287-290. http://www.ncbi.nlm.nih.gov/pubmed/10908026 Austin, B., and Austin, D. A. Bacterial fish pathogens: Disease of farmed and wild fish, sixth edition, 2016. doi:10.1007/978-3-31932674-0.

49. S.T. Chacko, S. T., S.T. Chandy, S. T., O.C. Abraham, O. C., et al. Pacemaker endocarditis caused by Pseudomonas aeruginosa treated successfully. J Assoc Physicians India. 2003; 51: 1021-1022.

http://www.ncbi.nlm.nih.gov/pubmed/14719599

50. Maskarinec, S. A., Thaden, J. T., Cyr, D. D., Ruffin, F., M. Souli, M., and Fowler, V.G. The
Risk of Cardiac Device-Related Infection in Bacteremic Patients Is Species Specific: Results of a 12-Year Prospective Cohort. Open Forum Infect Dis. 2017; 4: 132 doi:10.1093/ofid/ofx132 . 51. Oliva, A., Nguyen, B. L., Mascellino, M. T., et al. Sonication of explanted cardiac implants improves microbial detection in cardiac device infections. J Clin Microbiol. 2013;51(2):496-502 doi:10.1128/JCM.02230-12.

52. Da Costa, A., Kirkorian, G., Isaaz, K., and Touboul, P. [Secondary infections after pacemaker implantation]., La Rev Med Interne. 2000;21:256-265.

http://www.ncbi.nlm.nih.gov/pubmed/10763187

53. Mack, D., Rohde, H., Harris, L. G., Davies, A. P., Horstkotte, M. A., and Knobloch, J. K. Biofilm formation in medical device-related infection. Int J Artif Organs. 2006; 29 (4): 343359.

54. Veenstra, G. J., Cremers, F. F., van Dijk, H., and Fleer, A. Ultrastructural organization and regulation of a biomaterial adhesin of Staphylococcus epidermidis. J Bacteriol. 1996;178:537-541.

http://www.ncbi.nlm.nih.gov/pubmed/8550477

55. Vijaranakul, U., Nadakavukaren, M. J., Bayles, D. O., Wilkinson, B. J., and Jayaswal, R. K. Characterization of an $\mathrm{NaCl}$-sensitive Staphylococcus aureus mutant and rescue of the $\mathrm{NaCl}$-sensitive phenotype by glycine betaine but not by other compatible solutes. Appl Environ Microbiol.1997;63:1889-1897.

http://www.ncbi.nlm.nih.gov/pubmed/9143120

56. Vuong, C., and Otto, M. Staphylococcus epidermidis infections. Microbes Infect. 2002; 4: 481-489. http://www.ncbi.nlm.nih.gov/pubmed/11932199 Vanittanakom, N., Mack, D., and Götz, F. Molecular basis of intercellular adhesion in the biofilm-forming Staphylococcus epidermidis. Mol Microbiol.1996;20(5):1083-1091.

http://www.ncbi.nlm.nih.gov/pubmed/8809760

58. Oli, M. W., Otoo, H. N., Crowley, P. J., et al. Functional amyloid formation by Streptococcus mutans, Microbiol. (United Kingdom). 2012; 158: 2903-2916. doi:10.1099/mic.0.060855-0.

59. Araújo, A. M. M., de Oliveira, I. C. M., de Mattos, M. C., and Benchetrit, L. C. Cell surface hydrophobicity and adherence of a strain of group B streptococci during the post-antibiotic effect of penicillin. Rev Inst Med Trop Sao Paulo.2008;50(4):203-207. Hamadi, F., and Latrache, H. Comparison of contact angle measurement and microbial adhesion to solvents for assaying electron donor-electron acceptor (acid-base) properties of bacterial surface. Colloids Surf B Biointerfaces. 2008;65:134-139.

doi:10.1016/j.colsurfb.2008.03.010.

61. de M. Costa, G. F., Tognim, M. C. B., Cardoso, C. L., Carrara-Marrone, F. E., and Garcia, L. B. Preliminary evaluation of adherence on abiotic and cellular surfaces of Acinetobacter baumannii strains isolated from catheter tips. Braz J Infect Dis. 2006; 10: 346 - 351. http://www.ncbi.nlm.nih.gov/pubmed/17293924

62. Djeribi, R., Boucherit, Z., Bouchloukh, W., et al. A study of $\mathrm{pH}$ effects on the bacterial surface physicochemical properties of Acinetobacter baumannii. Colloids Surf B. Biointerfaces. 2013; 102:540-545.doi:10.1016/j.colsurfb.2012.08.047.

63. Pour, N. K. Dusane, D. H., Dhakephalkar, P. K., Zamin, F. R., Zinjarde, S. S., and Chopade, B. A. Biofilm formation by Acinetobacter baumannii strains isolated from urinary tract infection and urinary catheters. FEMS Immunol Med Microbiol. 2011;62: 328-338. 